\title{
Performance profiles of football teams in the UEFA \\ Champions League considering situational efficiency
}

\author{
Hongyou Liu ${ }^{1,2}$, Qing $\mathrm{Yi}^{3}$, Jesús-Vicente Giménez ${ }^{1}$, Miguel-Angel Gómez ${ }^{1}$ and Carlos \\ Lago-Peñas ${ }^{4}$ \\ ${ }^{1}$ Faculty of Physical Activity and Sport Sciences, Technical University of Madrid, Madrid, Spain \\ ${ }^{2}$ Sport Department, Shenzhen University, Shenzhen, China \\ ${ }^{3}$ Institute of Education, Hunan Agricultural University, Changsha, China \\ ${ }^{4}$ Faculty of Education and Sports Sciences, University of Vigo, Pontevedra, Spain
}

\begin{abstract}
Performance of football teams varies constantly due to the dynamic nature of this sport, whilst the typical performance and its spread can be represented by profiles combining different performance-related variables based on data from multiple matches. The current study aims to use a profiling technique to evaluate and compare match performance of football teams in the UEFA Champions League incorporating three situational variables (i.e. strength of team and opponent, match outcome and match location). Match statistics of 72 teams, 496 games across four seasons (2008-09 to 2012-13) of this competition were analysed. Sixteen performance-related events were included: shots, shots on target, shots from open play, shots from set piece, shots from counter attack, passes, pass accuracy (\%), crosses, through balls, corners, dribbles, possession, aerial success (\%), fouls, tackles, and yellow cards. Teams were classified into three levels of strength by a k-cluster analysis. Profiles of overall performance and profiles incorporating three situational variables for teams of all three levels of strength were set up by presenting the mean, standard deviation, median, lower and upper quartiles of the counts of each event to represent their typical performances and spreads. Means were compared by using one-way ANOVA and independent sample t test (for match location, home and away differences), and were plotted into the same radar charts after unifying all the event counts by standardised score. Established profiles can present straightforwardly typical performances of football teams of different levels playing in different situations, which could provide detailed references for coaches and analysts to evaluate performances of upcoming opposition and of their own.
\end{abstract}

Keywords: soccer, notational analysis, match statistics, performance indicators 


\section{Introduction}

Profiles in sports performance analysis can be defined as collections and combinations of valid and reliable typical performance indicators within analysed sports brought together to represent the performance of an athlete or/and a team (O'Donoghue, 2013). Performance profiles can represent players' or/and teams' typical performance and its spread by using performance-related variables based on data from multiple matches (Hughes et al., 2001; O'Donoghue, 2005; 2013).

In recent years, researchers made great efforts to develop meaningful and useful performance profiles in sport (Butterworth et al., 2013; Eugster, 2012; Hughes et al., 2001; James et al., 2005; O'Donoghue, 2005; 2013). The main profiling techniques were discussed and compared by O'Donoghue (2013). Specifically, the profiling technique of using median and 95\% confidence intervals (James et al., 2005) was believed to be better in comparing differences between performers and the technique of using median and quantiles (O'Donoghue, 2005) was believed to be better in representing typical performance and its spread of single performer. However, based on a sample of large size, a combination of these two profiling techniques can be extended to present and compare the performance of various performers by taking the mean of the counts of each performance-related variable of the performers.

The highly complex and dynamic nature of football makes the performance profiling within this sport difficult (Vilar et al., 2012). However, based on sufficient match observations, the typical performances of football teams and their spreads and variations can be represented by profiles taking the mean, upper and lower quantiles and standard deviations of the counts of different validated performance-related match events (Hughes et al., 2001; O'Donoghue, 2005; 2013).

In addition, although the profiles can interpret variations in teams' performances by representing the random variability in event count values, the variation associated to effects of situational variables (e.g. level of team/opposition, match outcome, match location) cannot be incorporated (Taylor et al., 2010). Nevertheless, it has been found that both physical and technical-tactical performances of football are influenced by situational conditions at a behavioural level (Gómez et al., 2013; Lago-Peñas, 2012; Taylor et al., 2010). These situational conditions mainly include match location (i.e. play at home/away), match status/outcome (i.e. winning/win, losing/loss or drawing/draw), strength of team and opponent, type of match (e.g. league/cup, pre-season/final of competition), match period (e.g. first half/second half), and so on (Gómez et al., 2013; Lago-Peñas, 2012; Mackenzie and Cushion, 2013; Sarmento et al., 2014; Taylor et al., 2010; Taylor et al., 2008).

Influences of aforementioned situational variables on technical and tactical performance of football teams were evaluated previously by the variation of counts of technical match actions which include variables related to scoring, such as shots; variables related to attacking and passing, such as passes; and variables related to 
defending, such as tackles (Castellano et al., 2012; Lago-Peñas and Lago-Ballesteros, 2011; Lago-Peñas et al., 2010; Lago-Peñas et al., 2011). Prior studies compared performances of different levels of team from Spanish First Division (Lago-Peñas et al., 2010), English Premier League (Oberstone, 2009) and Greek Football First League (Armatas et al., 2009) and found that variables related to goal scoring, passing and attacking differentiate due to the strength of team. While the variation of possession strategies (Lago, 2009), aerial challenges, dribbles, and passes (Taylor et $a l ., 2008)$ was found to be associated with the strength of the opponent. Studies in Spanish First Division (Lago-Peñas et al., 2010), World Cup (Castellano et al., 2012) and Group Stage of UEFA Champions League (Lago-Peñas et al., 2011) showed that various performance-related match variables were found different across won, drawn and lost matches. Meanwhile, studies of Spanish First Division (Gómez et al., 2012; Lago-Peñas and Lago-Ballesteros, 2011; Lago, 2009) and England Premier League (Taylor et al., 2010; Taylor et al., 2008) showed that almost all match variables differentiated when teams played at home from when playing away. Therefore, it could be argued that situational variables of strength of team and opponent, match outcome and match location should be incorporated into performance profiles of football teams.

Based on the framework discussed above, the current study intends to establish technical and tactical performance profiles of football teams in the UEFA Champions League incorporating three situational variables: strength of team and opponent, match outcome and match location.

\section{Method}

\subsection{Sample}

The UEFA Champions League is an annual transnational club competition that is only disputed by the best ranked clubs in the previous season of domestic football leagues from the Union of European Football Associations (UEFA). It consists by three qualifying rounds, a group stage and a knockout stage. After qualifying rounds, 32 clubs enter the group stage where clubs are divided into 8 groups of four clubs, and every club plays at home and away against each of its group opponents. Winners and runners-up of each group qualify to the knockout stage in which a winner from one group plays against a runner-up from another group and teams from the same national association will not be drawn against each other. While from quarter-finals onwards, the draw will be totally random. Knock-out ties are played in a two-legged format: two teams play against each at home and away, and aggregate score of the two matches decides who reaches the next round. However, the final is always a single match held in a neutral field. All together, in each season, the UEFA Champions League consists of 125 matches: 96 at the group stage (12 matches in every group) and 29 matches $(16+8+4+1)$ at the knock-out stage. More detailed description 
about the introduction and competing procedure of this tournament can be found in Lago-Peñas et al. (2011, pp. 139-140).

All the 500 matches of group stage and knockout stage of UEFA Champions League in the seasons of 2009-10, 2010-11, 2011-12 and 2012-13 were chosen as the sample of the current study. However, the 4 matches in which extra time was played were excluded, which made the final sample to 496 matches ( $n=992$ observations).

\subsection{Reliability of Data}

The data employed in the current study were collected from the public accessed website "whoscored.com" whose data recourse is OPTA Sportsdata Company. The reliability of the tracking system (OPTA Client System) which is used by the company to collect football match statistics has been verified by Liu and colleagues (Liu et al., 2013) which showed that team match events coded by independent operators using this system reached a very good agreement (weighted kappa values were 0.92 and 0.94). Ethics committee approval of the study was obtained from the local university.

\subsection{Variables}

As discussed in the introduction, based on the review of available literature (Castellano et al., 2012; Lago-Peñas and Lago-Ballesteros, 2011; Lago-Peñas et al., 2010; Lago-Peñas et al., 2011; Liu et al., 2013), three groups of performance-related match events were chosen: (1) variables related to scoring: shots, shots on target, shots from open play, shots from set piece and shots from counter attack; (2) variables related to attacking and passing: passes, pass accuracy (\%), crosses, through balls, corners, dribbles, possession, aerial success (\%); and (3) variables related to defending: fouls, tackles, yellow cards. Operational definitions of the variables can be found elsewhere (Liu et al., 2013).

Three situational variables were also included: (1) strength of team and opponent; (2) match outcome (win, draw and loss); and (3) match location (home and away).

\subsection{Identification of Close Game}

As being stated by research in basketball (Sampaio et al., 2010) and water polo (Gómez et al., 2014; Lupo et al., 2014), specific winning and losing margin should be employed to identify the closeness of a game, and only close games can represent the best performance between the confronting two teams (Gómez et al., 2014). Therefore, a k-means cluster analysis were undertook to identify the cut-off value in goal differences in order to classify close games and unbalanced games (Gómez et al., 2014; Lupo et al., 2014; Sampaio et al., 2010). Results identified one cluster of 96 games (unbalanced games) with a goal difference of more than and equal to 3 goals ( $3.63 \pm 0.97$, ranged from 3 to $7, \mathrm{n}=192$ observations), and another cluster of 400 games (close games) with a difference of less than 3 goals $(1 \pm 0.75$, ranged from 0 to $2, n=800$ observations). Only close games were included in the statistical analysis. 


\subsection{Classification of Team Strength}

UEFA season club coefficients were used to classify the strength of team. The season club coefficient is a point system based on results of clubs competing in the current season of UEFA Champions League and UEFA Europa League. Detailed information about the point system can be found at the official website of UEFA (UEFA, 2013).

The strength of team was classified into three groups using the method of k-means cluster analysis: (1) high-level teams (UEFA season club coefficients: $31.77 \pm 3.19$, rang: 26.67 to $36.67,12$ teams, $n=180$ observations); (2) intermediate-level teams (UEFA season club coefficients: $21.21 \pm 2.59$, rang: 16.05 to $26.02,39$ teams, $\mathrm{n}=388$ observations); and (3) low-level teams (UEFA season club coefficients: $9.38 \pm 2.69$, rang: 4.55 to $15.23,39$ teams, $\mathrm{n}=232$ observations) (Gómez et al., 2013; Marcelino et al., 2011).

\subsection{Development of Performance Profiles}

As discussed by O'Donoghue (2005), the mean, standard deviation (SD), median, lower and upper quartiles of the count values of performance-related variables can be used to represent players'/teams' typical performances, variations and spreads, because the spread of $50 \%$ of values can be represented by the mean and 0.674 SD of the normally distributed data and by the median, lower and upper quartiles of the non-normally distributed data. Therefore, profiles of overall performances and profiles incorporating three situational variables (strength of opponent, match outcome and match location) for teams of all three levels of strength were set up by presenting the means \pm SDs, medians, lower and upper quartiles of the counts of each performance-related variable to represent their typical performances, variations and spreads. Meanwhile, their means were compared by using one-way ANOVA and independent sample $t$ test (for match location, home and away differences).

Furthermore, the counts of all the performance-related match variables of all teams were transferred into standardised score (Z-Score, $Z$ ), and were unified into the same scale using the formulation " $T=20 Z+50 "$ (Barriopedro and Muniesa, 2012). Therefore, means, medians, lower and upper quartiles of all variables of all three levels of team can be plotted into the same radar chart to be compared. The current study plotted only the transferred scores of means of teams of different levels of strength, and means of teams of different levels of strength playing in different situational conditions.

The k-means cluster analysis, one-way ANOVA and independent sample t test were all performed in the data package of IBM SPSS Statistics for Windows, Version 20.0 (Armonk, NY: IBM Corp.). Significant levels of the later two tests were both set to $p<0.05$. Effect size of one-way ANOVA was estimated by the partial eta-squared $\left(\eta_{\mathrm{p}}{ }^{2}\right)$, and the scale was: 0.01 small, 0.06 medium, 0.14 large (Cohen, 1988). Effect size of comparing home and away differences was estimated by the spreadsheet of Hopkins 
(2007). Magnitudes were assessed as: 0-0.2 trivial, 0.2-0.6 small, 0.6-1.2 moderate, 1.2-2.0 large, and >2.0 very large (Hopkins et al., 2009).

\section{Result and Discussion}

\subsection{Overall performance of different levels of team}

The typical performances and their statistical significances and spreads of teams of different levels of strength were presented in Table 1. Transferred scores of means were plotted in Figure 1. 
Table1. Profiles of overall performance of different levels of teams

\begin{tabular}{|c|c|c|c|c|c|c|c|c|c|c|c|c|c|c|c|c|}
\hline \multirow[b]{2}{*}{ Variables } & \multicolumn{4}{|c|}{ High-level teams } & \multicolumn{4}{|c|}{ Intermediate-level teams } & \multicolumn{4}{|c|}{ Low-level teams } & \multicolumn{4}{|c|}{ ANOVA } \\
\hline & Mean \pm SD & Median & $\begin{array}{c}\text { Lower } \\
\text { Quartile }\end{array}$ & $\begin{array}{c}\text { Upper } \\
\text { Quartile }\end{array}$ & Mean \pm SD & Median & $\begin{array}{c}\text { Lower } \\
\text { Quartile }\end{array}$ & $\begin{array}{c}\text { Upper } \\
\text { Quartile }\end{array}$ & Mean \pm SD & Median & $\begin{array}{c}\text { Lower } \\
\text { Quartile }\end{array}$ & $\begin{array}{c}\text { Upper } \\
\text { Quartile }\end{array}$ & df & $\mathbf{F}$ & Sig. & $\eta_{p}^{2}$ \\
\hline Shots & $15.4 \pm 6.1$ & 15 & 11 & 19 & $13.8 \pm 5.9$ & 13 & 9 & 17 & $11.9 \pm 5.8$ & 11 & 8 & 15 & 2,789 & 17.720 & $* * *$ & 0.043 \\
\hline SoT & $5.8 \pm 2.8$ & 6 & 4 & 7 & $4.7 \pm 2.5$ & 4 & 3 & 6 & $3.9 \pm 2.4$ & 3 & 2 & 5 & 2,789 & 28.729 & $* * *$ & 0.068 \\
\hline SfOP & $9.7 \pm 5.0$ & 9 & 6 & 12 & $8.6 \pm 4.4$ & 8 & 5 & 11 & $7.6 \pm 4.5$ & 7 & 4 & 10 & 2,789 & 11.150 & $* * *$ & 0.027 \\
\hline SfSP & $3.4 \pm 2.2$ & 3 & 2 & 5 & $3.0 \pm 2.1$ & 3 & 2 & 4 & $2.7 \pm 2.0$ & 2 & 1 & 4 & 2,789 & 7.383 & $* * *$ & 0.018 \\
\hline SfCA & $0.5 \pm 0.8$ & 0 & 0 & 1 & $0.5 \pm 0.8$ & 0 & 0 & 1 & $0.4 \pm 0.7$ & 0 & 0 & 1 & 2,789 & 2.836 & & 0.007 \\
\hline Passes & $539.0 \pm 169.5$ & 527 & 404 & 650 & $479.6 \pm 110.6$ & 473 & 404 & 557 & $437.9 \pm 100.9$ & 431 & 368 & 498 & 2,789 & 31.360 & $* * *$ & 0.074 \\
\hline PA & $81.4 \pm 7.5$ & 82 & 77 & 88 & $79.1 \pm 6.4$ & 80 & 75 & 84 & $76.9 \pm 7.2$ & 77 & 73 & 82 & 2,789 & 20.098 & $* * *$ & 0.048 \\
\hline Crosses & $20.3 \pm 9.1$ & 20 & 14 & 25 & $21.3 \pm 8.9$ & 20 & 15 & 27 & $19.6 \pm 9.4$ & 19 & 13 & 26 & 2,789 & 2.368 & & 0.006 \\
\hline $\mathrm{TB}$ & $5.0 \pm 4.3$ & 4 & 2 & 7 & $3.2 \pm 2.8$ & 3 & 1 & 5 & $2.4 \pm 2.4$ & 2 & 1 & 3 & 2,789 & 37.735 & $* * *$ & 0.087 \\
\hline Corners & $5.5 \pm 3.0$ & 5 & 4 & 7 & $5.2 \pm 3.0$ & 5 & 3 & 7 & $4.7 \pm 2.8$ & 4 & 3 & 6 & 2,789 & 4.115 & $*$ & 0.010 \\
\hline Dribbles & $9.1 \pm 4.2$ & 9 & 6 & 11 & $8.1 \pm 4.0$ & 8 & 5 & 10 & $6.7 \pm 3.6$ & 6 & 4 & 9 & 2,789 & 17.420 & $* * *$ & 0.042 \\
\hline Possession & $54.4 \pm 14.5$ & 55 & 45 & 65 & $50.4 \pm 10.6$ & 51 & 43 & 58 & $45.8 \pm 10.9$ & 46 & 38 & 53 & 2,789 & 26.293 & $* * *$ & 0.062 \\
\hline AS & $52.2 \pm 14.6$ & 51 & 44 & 63 & $50.7 \pm 14.0$ & 50 & 41 & 60 & $47.2 \pm 15.5$ & 46 & 36 & 57 & 2,789 & 6.941 & $* *$ & 0.017 \\
\hline Fouls & $13.7 \pm 4.6$ & 13 & 10 & 17 & $14.5 \pm 4.2$ & 14 & 12 & 17 & $15.2 \pm 4.5$ & 15 & 12 & 18 & 2,789 & 5.708 & $* *$ & 0.014 \\
\hline Tackles & $20.9 \pm 6.1$ & 21 & 17 & 25 & $21.3 \pm 6.0$ & 21 & 17 & 25 & $21.1 \pm 5.9$ & 21 & 17 & 25 & 2,789 & 0.260 & & 0.001 \\
\hline $\mathrm{YC}$ & $1.9 \pm 1.3$ & 2 & 1 & 3 & $1.9 \pm 1.2$ & 2 & 1 & 3 & $2.1 \pm 1.2$ & 2 & 1 & 3 & 2,789 & 2.517 & & 0.006 \\
\hline
\end{tabular}

Note 1. SoT =Shots on Target; SfOP =Shots from Open Play; SfSP =Shots from Set Piece; SfCA =Shots from Counter Attack; PA =Pass Accuracy (\%); TB =Through Balls; AS $=$ Aerial Success $(\%)$; YC $=$ Yellow Cards

Note $2 . * p<0.05, * * p<0.01$, $* * * p<0.001$

Note 3. Abbreviations are applicable to the whole text 


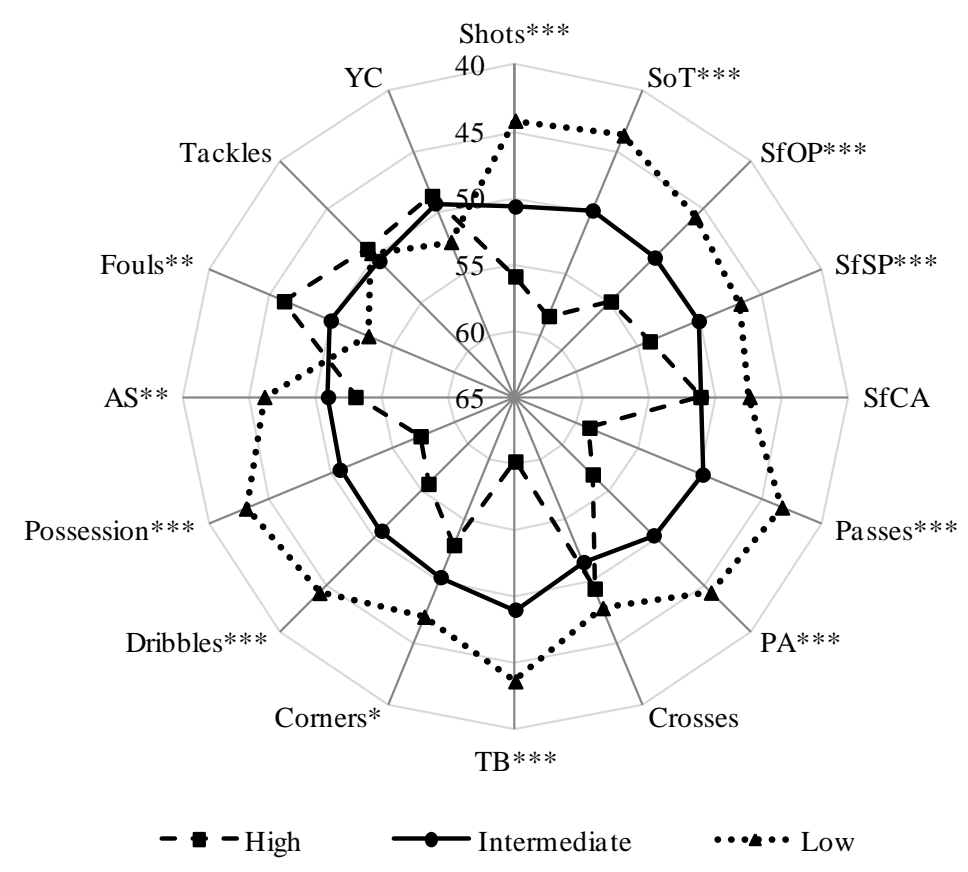

Figure 1. Comparison on overall performance of different levels of team

As shown in profiles of overall performance of high, intermediate and low level teams, there were performance-related variables from all the three groups that significantly distinguished from different levels of teams in the UEFA Champions League. This finding is different from prior research on the comparison of performance of different levels of teams from Spanish First Division (Lago-Peñas et al., 2010), English Premier League (Oberstone, 2009) and Greek Football First League (Armatas et al., 2009) which found that differences between high-level teams and low-level teams mainly existed in the variables related to goal scoring, passing and attacking. Differences in the result may be explained by the fact that the UEFA Champions League is more competitive than national leagues on which other studies were conducted.

\subsection{Performance profiles when facing different levels of opposition}

Performance profiles of high, intermediate and low level teams when facing different levels of opposition were displayed in Table 2. Statistical significances were also shown in the table. Comparison on the mean differences can be seen from Figure 2 as well. 
Table 2. Profiles of performance of high, intermediate and low-level teams when facing different levels of opposition

\begin{tabular}{|c|c|c|c|c|c|c|c|c|c|c|c|c|c|c|c|c|c|c|c|c|c|}
\hline \multirow{3}{*}{ Variables } & \multicolumn{7}{|c|}{ High-level teams } & \multicolumn{7}{|c|}{ Intermediate-level teams } & \multicolumn{7}{|c|}{ Low-level teams } \\
\hline & \multirow{2}{*}{$\begin{array}{c}\text { vs. High } \\
\text { Mean } \pm \text { SD }\end{array}$} & \multirow{2}{*}{$\begin{array}{l}\text { vs. Interm. } \\
\text { Mean } \pm \text { SD }\end{array}$} & \multirow{2}{*}{$\begin{array}{c}\text { vs. Low } \\
\text { Mean } \pm \text { SD }\end{array}$} & \multicolumn{4}{|c|}{ ANOVA } & \multirow{2}{*}{$\begin{array}{c}\text { vs. High } \\
\text { Mean } \pm \text { SD }\end{array}$} & \multirow{2}{*}{$\begin{array}{l}\text { vs. Interm. } \\
\text { Mean } \pm \text { SD }\end{array}$} & \multirow{2}{*}{$\begin{array}{c}\text { vs. Low } \\
\text { Mean } \pm \text { SD }\end{array}$} & \multirow[b]{2}{*}{ df } & \multicolumn{3}{|c|}{ ANOVA } & \multirow{2}{*}{$\begin{array}{c}\text { vs. High } \\
\text { Mean } \pm \text { SD }\end{array}$} & \multirow{2}{*}{$\begin{array}{l}\text { vs. Interm. } \\
\text { Mean } \pm \text { SD }\end{array}$} & \multirow{2}{*}{$\begin{array}{c}\text { vs. Low } \\
\text { Mean } \pm \text { SD }\end{array}$} & \multicolumn{4}{|c|}{ ANOVA } \\
\hline & & & & df & $\mathbf{F}$ & Sig. & $\eta_{p}^{2}$ & & & & & $\mathbf{F}$ & Sig. & $\eta_{p}{ }^{2}$ & & & & df & $\mathbf{F}$ & Sig. & $\eta_{p}^{2}$ \\
\hline Shots & $12.9 \pm 5.7$ & $15.5 \pm 5.8$ & $18.7 \pm 5.9$ & 2,176 & 11.155 & $* * *$ & 0.113 & $11.8 \pm 5.1$ & $13.9 \pm 5.6$ & $15.1 \pm 6.5$ & 2,382 & 8.633 & $* * *$ & 0.043 & $8.8 \pm 3.9$ & $12.1 \pm 5.7$ & $13.5 \pm 6.5$ & 2,225 & 7.420 & $* * *$ & 0.062 \\
\hline SoT & $4.7 \pm 2.7$ & $6.1 \pm 2.7$ & $6.4 \pm 2.8$ & 2,176 & 5.736 & $* *$ & 0.061 & $3.9 \pm 2.3$ & $4.6 \pm 2.5$ & $5.2 \pm 2.4$ & 2,382 & 6.880 & $* *$ & 0.035 & $2.8 \pm 1.9$ & $4.1 \pm 2.4$ & $4.1 \pm 2.4$ & 2,225 & 4.583 & * & 0.039 \\
\hline SfOP & $8.3 \pm 4.7$ & $9.7 \pm 4.8$ & $11.7 \pm 5.6$ & 2,176 & 5.579 & $* *$ & 0.060 & $7.1 \pm 3.9$ & $8.7 \pm 4.3$ & $9.6 \pm 4.6$ & 2,382 & 8.690 & $* * *$ & 0.044 & $5.3 \pm 3.0$ & $7.8 \pm 4.3$ & $8.7 \pm 5.2$ & 2,225 & 7.383 & $* * *$ & 0.062 \\
\hline SfSP & $2.9 \pm 2.1$ & $3.6 \pm 2.3$ & $3.8 \pm 1.7$ & 2,176 & 2.596 & & 0.029 & $3.0 \pm 1.9$ & $3.0 \pm 2.2$ & $3.1 \pm 2.2$ & 2,382 & 0.414 & & 0.002 & $2.2 \pm 1.7$ & $2.6 \pm 2.0$ & $3.1 \pm 2.2$ & 2,225 & 2.616 & & 0.023 \\
\hline SfCA & $0.5 \pm 0.7$ & $0.5 \pm 0.8$ & $0.7 \pm 1.0$ & 2,176 & 0.692 & & 0.008 & $0.3 \pm 0.6$ & $0.6 \pm 0.9$ & $0.6 \pm 0.9$ & 2,382 & 3.910 & * & 0.020 & $0.4 \pm 0.7$ & $0.4 \pm 0.7$ & $0.5 \pm 0.7$ & 2,225 & 0.447 & & 0.004 \\
\hline Passes & $491.8 \pm 197.1$ & $535.4 \pm 142.6$ & $612.7 \pm 163.7$ & 2,176 & 5.280 & $* *$ & 0.057 & $437.9 \pm 100.8$ & $473.2 \pm 101.2$ & $516.5 \pm 117.4$ & 2,382 & 14.101 & $* * *$ & 0.069 & $405.4 \pm 109.7$ & $432.5 \pm 91.2$ & $470.7 \pm 107.6$ & 2,225 & 5.134 & $* *$ & 0.044 \\
\hline $\mathrm{PA}$ & $78.8 \pm 9.4$ & $81.7 \pm 6.3$ & $84.3 \pm 5.8$ & 2,176 & 6.114 & $* *$ & 0.065 & $77.4 \pm 7.0$ & $78.9 \pm 5.9$ & $80.4 \pm 6.3$ & 2,382 & 5.785 & $* *$ & 0.029 & $76.4 \pm 6.7$ & $76.7 \pm 7.3$ & $77.8 \pm 7.2$ & 2,225 & 0.576 & & 0.005 \\
\hline Crosses & $17.6 \pm 8.8$ & $20.2 \pm 8.7$ & $24.2 \pm 8.9$ & 2,176 & 7.112 & $* * *$ & 0.075 & $20.1 \pm 8.3$ & $21.5 \pm 8.7$ & $21.9 \pm 9.5$ & 2,382 & 1.125 & & 0.006 & $14.9 \pm 7.5$ & $19.9 \pm 9.4$ & $22.0 \pm 9.4$ & 2,225 & 6.620 & $* *$ & 0.056 \\
\hline TB & $4.9 \pm 3.8$ & $4.8 \pm 4.7$ & $5.7 \pm 3.9$ & 2,176 & 0.339 & & 0.004 & $3.1 \pm 2.5$ & $3.1 \pm 2.9$ & $3.5 \pm 2.9$ & 2,382 & 1.075 & & 0.006 & $2.3 \pm 1.8$ & $2.4 \pm 2.5$ & $2.4 \pm 2.5$ & 2,225 & 0.009 & & 0.000 \\
\hline Corners & $4.8 \pm 2.8$ & $5.6 \pm 3.2$ & $6.5 \pm 2.9$ & 2,176 & 3.804 & $*$ & 0.041 & $4.7 \pm 2.8$ & $5.1 \pm 2.7$ & $5.5 \pm 3.3$ & 2,382 & 2.123 & & 0.011 & $3.5 \pm 2.6$ & $4.8 \pm 2.9$ & $5.1 \pm 2.7$ & 2,225 & 3.626 & $*$ & 0.031 \\
\hline Dribbles & $8.9 \pm 4.5$ & $8.5 \pm 3.0$ & $10.5 \pm 5.6$ & 2,176 & 2.743 & & 0.030 & $7.2 \pm 3.8$ & $8.2 \pm 3.6$ & $8.5 \pm 4.5$ & 2,382 & 3.080 & * & 0.016 & $6.1 \pm 3.4$ & $6.4 \pm 3.4$ & $7.9 \pm 3.9$ & 2,225 & 4.252 & * & 0.036 \\
\hline Possession & $50.0 \pm 19.2$ & $54.6 \pm 10.8$ & $60.1 \pm 12.1$ & 2,176 & 5.272 & $* *$ & 0.057 & $45.4 \pm 10.8$ & $50.0 \pm 9.8$ & $54.3 \pm 9.9$ & 2,382 & 20.576 & $* * *$ & 0.097 & $39.9 \pm 12.1$ & $45.7 \pm 9.9$ & $50.0 \pm 10.6$ & 2,225 & 9.981 & $* * *$ & 0.081 \\
\hline AS & $50.0 \pm 13.5$ & $50.6 \pm 14.7$ & $58.7 \pm 14.1$ & 2,176 & 5.582 & $* *$ & 0.060 & $49.4 \pm 14.7$ & $50.0 \pm 12.7$ & $52.5 \pm 14.9$ & 2,382 & 1.653 & & 0.009 & $41.3 \pm 14.1$ & $47.6 \pm 14.9$ & $50.0 \pm 16.9$ & 2,225 & 4.201 & $*$ & 0.036 \\
\hline Fouls & $14.8 \pm 4.8$ & $13.6 \pm 4.2$ & $12.2 \pm 4.7$ & 2,176 & 3.121 & $*$ & 0.034 & $15.3 \pm 4.6$ & $14.4 \pm 4.2$ & $14.1 \pm 3.9$ & 2,382 & 2.365 & & 0.012 & $14.0 \pm 4.5$ & $15.8 \pm 4.8$ & $14.7 \pm 3.7$ & 2,225 & 2.719 & & 0.024 \\
\hline Tackles & $21.7 \pm 7.4$ & $21.0 \pm 5.7$ & $19.8 \pm 4.9$ & 2,176 & 0.901 & & 0.010 & $20.7 \pm 5.4$ & $22.8 \pm 5.9$ & $19.9 \pm 6.1$ & 2,382 & 9.541 & $* * *$ & 0.048 & $22.5 \pm 5.2$ & $21.1 \pm 6.1$ & $20.1 \pm 5.8$ & 2,225 & 1.900 & & 0.017 \\
\hline $\mathrm{YC}$ & $2.3 \pm 1.5$ & $1.8 \pm 1.2$ & $1.5 \pm 1.4$ & 2,176 & 4.849 & $* *$ & 0.052 & $2.3 \pm 1.3$ & $1.9 \pm 1.2$ & $1.6 \pm 1.2$ & 2,382 & 8.183 & $* * *$ & 0.041 & $2.3 \pm 1.2$ & $2.1 \pm 1.3$ & $2.1 \pm 1.2$ & 2,225 & 0.509 & & 0.005 \\
\hline
\end{tabular}

Note. Median, lower and upper quartiles were removed due to table size limitation 

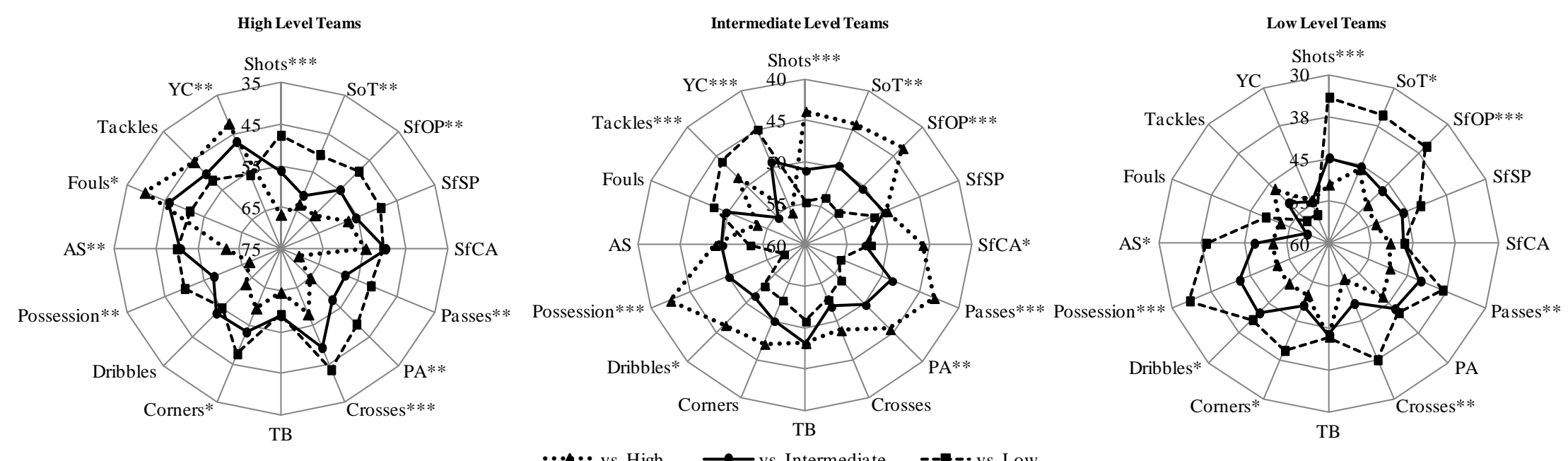

Figure 2. Comparison on performances of high, intermediate and low level teams when facing different qualities of oppositions 
Performance profiles of teams when facing different levels of opposition identified that in the UEFA Champions League, variables of shots, shots on target, shots from open play, passes, ball possession, aerial success and fouls varied for all three levels of team when facing different levels of opposition. Whilst previous studies in a professional Spanish football team (Lago, 2009) and a single professional British football team (Taylor et al., 2008) found that possession strategies, aerial challenges, dribbles, and passes were influenced by level of opposition. Results also showed that playing against opposition with higher strength demands higher level of technical and tactical performance.

\subsection{Performance profiles when ending with different match outcomes}

Table 3 showed profiles of performance of high, intermediate and low level teams when ending with loss, draw and win. Figure 3 plotted the transferred scores of means of each performance variable of each level of team ending with different match results. 
Table 3. Profiles of performance of high, intermediate and low-level teams when ending with different match outcomes

\begin{tabular}{|c|c|c|c|c|c|c|c|c|c|c|c|c|c|c|c|c|c|c|c|c|c|}
\hline \multirow{3}{*}{ Variables } & \multicolumn{7}{|c|}{ High-level teams } & \multicolumn{7}{|c|}{ Intermediate-level teams } & \multicolumn{7}{|c|}{ Low-level teams } \\
\hline & \multirow{2}{*}{$\begin{array}{c}\text { Loss } \\
\text { Mean } \pm \text { SD }\end{array}$} & \multirow{2}{*}{$\begin{array}{c}\text { Draw } \\
\text { Mean } \pm \text { SD }\end{array}$} & \multirow{2}{*}{$\begin{array}{c}\text { Win } \\
\text { Mean } \pm \text { SD }\end{array}$} & \multicolumn{4}{|c|}{ ANOVA } & \multirow{2}{*}{$\begin{array}{c}\text { Loss } \\
\text { Mean } \pm \text { SD }\end{array}$} & \multirow{2}{*}{$\begin{array}{c}\text { Draw } \\
\text { Mean } \pm \text { SD }\end{array}$} & \multirow{2}{*}{$\begin{array}{c}\text { Win } \\
\text { Mean } \pm \text { SD }\end{array}$} & \multicolumn{4}{|c|}{ ANOVA } & \multirow{2}{*}{$\begin{array}{c}\text { Loss } \\
\text { Mean } \pm \text { SD }\end{array}$} & \multirow{2}{*}{$\begin{array}{c}\text { Draw } \\
\text { Mean } \pm \text { SD }\end{array}$} & \multirow{2}{*}{$\begin{array}{c}\text { Win } \\
\text { Mean } \pm \text { SD }\end{array}$} & \multicolumn{4}{|c|}{ ANOVA } \\
\hline & & & & df & $\mathbf{F}$ & Sig. & $\eta_{p}{ }^{2}$ & & & & df & $\mathbf{F}$ & Sig. & $\eta_{p}{ }^{2}$ & & & & df & $\mathbf{F}$ & Sig. & $\eta_{p}{ }^{2}$ \\
\hline Shots & $12.9 \pm 6.8$ & $15.3 \pm 5.1$ & $16.6 \pm 6.0$ & 2,176 & 5.081 & $* *$ & 0.055 & $12.9 \pm 5.6$ & $14.0 \pm 6.3$ & $14.5 \pm 5.8$ & 2,382 & 2.538 & & 0.013 & $11.3 \pm 5.5$ & $12.8 \pm 6.2$ & $12.4 \pm 6.0$ & 2,225 & 1.434 & & 0.013 \\
\hline SoT & $4.5 \pm 2.6$ & $5.4 \pm 2.5$ & $6.5 \pm 2.9$ & 2,176 & 8.309 & $* * *$ & 0.086 & $3.8 \pm 2.3$ & $4.5 \pm 2.4$ & $5.4 \pm 2.4$ & 2,382 & 16.045 & $* * *$ & 0.077 & $3.3 \pm 2.0$ & $4.4 \pm 2.5$ & $4.6 \pm 2.7$ & 2,225 & 7.250 & **** & 0.061 \\
\hline SfOP & $8.7 \pm 5.3$ & $9.3 \pm 4.4$ & $10.3 \pm 5.2$ & 2,176 & 1.567 & & 0.017 & $7.8 \pm 4.4$ & $8.9 \pm 4.4$ & $9.0 \pm 4.3$ & 2,382 & 3.491 & * & 0.018 & $7.3 \pm 4.2$ & $8.1 \pm 4.8$ & $7.9 \pm 4.8$ & 2,225 & 0.835 & & 0.007 \\
\hline SfSP & $2.7 \pm 1.9$ & $3.5 \pm 2.1$ & $3.8 \pm 2.2$ & 2,176 & 3.700 & $*$ & 0.040 & $2.9 \pm 1.9$ & $3.2 \pm 2.4$ & $3.1 \pm 2.0$ & 2,382 & 0.360 & & 0.002 & $2.7 \pm 1.9$ & $2.7 \pm 2.3$ & $2.6 \pm 1.8$ & 2,225 & 0.099 & & 0.001 \\
\hline SfCA & $0.4 \pm 0.7$ & $0.5 \pm 0.7$ & $0.6 \pm 0.9$ & 2,176 & 1.482 & & 0.017 & $0.3 \pm 0.7$ & $0.5 \pm 0.8$ & $0.8 \pm 0.9$ & 2,382 & 10.846 & $* * *$ & 0.054 & $0.3 \pm 0.6$ & $0.5 \pm 0.8$ & $0.7 \pm 0.7$ & 2,225 & 4.998 & $* *$ & 0.043 \\
\hline Passes & $506.9 \pm 186.5$ & $530.6 \pm 159.2$ & $556.8 \pm 166.8$ & 2,176 & 1.115 & & 0.013 & $465.6 \pm 99.5$ & $470.7 \pm 102.6$ & $497.0 \pm 122.0$ & 2,382 & 3.099 & * & 0.016 & $435.8 \pm 95.6$ & $445.1 \pm 104.8$ & $431.9 \pm 112.4$ & 2,225 & 0.236 & & 0.002 \\
\hline PA & $79.4 \pm 8.9$ & $81.1 \pm 7.1$ & $82.3 \pm 6.9$ & 2,176 & 1.971 & & 0.022 & $78.3 \pm 6.5$ & $79.0 \pm 5.7$ & $79.8 \pm 6.7$ & 2,382 & 1.917 & & 0.010 & $77.1 \pm 6.2$ & $76.8 \pm 8.9$ & $76.6 \pm 6.8$ & 2,225 & 0.112 & & 0.001 \\
\hline Crosses & $19.9 \pm 10.6$ & $19.9 \pm 8.6$ & $20.7 \pm 8.7$ & 2,176 & 0.211 & & 0.002 & $21.9 \pm 9.4$ & $21.1 \pm 9.9$ & $20.9 \pm 7.8$ & 2,382 & 0.464 & & 0.002 & $19.6 \pm 9.8$ & $20.7 \pm 8.5$ & $17.5 \pm 9.0$ & 2,225 & 1.423 & & 0.012 \\
\hline ТВ & $4.1 \pm 3.9$ & $5.1 \pm 3.9$ & $5.4 \pm 4.6$ & 2,176 & 1.284 & & 0.014 & $2.8 \pm 2.4$ & $3.6 \pm 3.0$ & $3.3 \pm 2.9$ & 2,382 & 2.303 & & 0.012 & $2.1 \pm 1.9$ & $2.9 \pm 2.9$ & $2.5 \pm 2.6$ & 2,225 & 2.674 & & 0.023 \\
\hline Corners & $5.1 \pm 3.3$ & $5.2 \pm 3.0$ & $5.9 \pm 2.9$ & 2,176 & 1.524 & & 0.017 & $5.0 \pm 2.8$ & $5.4 \pm 3.3$ & $5.1 \pm 2.9$ & 2,382 & 0.460 & & 0.002 & $4.6 \pm 3.0$ & $5.0 \pm 2.7$ & $4.4 \pm 2.6$ & 2,225 & 0.649 & & 0.006 \\
\hline Dribbles & $8.0 \pm 4.3$ & $9.1 \pm 3.8$ & $9.5 \pm 4.3$ & 2,176 & 1.720 & & 0.019 & $7.8 \pm 3.7$ & $7.5 \pm 3.6$ & $8.7 \pm 4.3$ & 2,382 & 3.067 & * & 0.016 & $6.3 \pm 3.6$ & $7.3 \pm 3.5$ & $7.1 \pm 3.7$ & 2,225 & 1.598 & & 0.014 \\
\hline Possession & $51.1 \pm 17.0$ & $53.2 \pm 14.8$ & $56.3 \pm 12.9$ & 2,176 & 1.831 & & 0.020 & $49.0 \pm 10.6$ & $50.1 \pm 10.1$ & $51.8 \pm 10.7$ & 2,382 & 2.551 & & 0.013 & $45.3 \pm 11.1$ & $47.6 \pm 10.7$ & $44.5 \pm 10.7$ & 2,225 & 1.261 & & 0.011 \\
\hline AS & $52.7 \pm 15.5$ & $52.7 \pm 15.7$ & $51.7 \pm 13.7$ & 2,176 & 0.096 & & 0.001 & $51.2 \pm 13.5$ & $50.4 \pm 14.7$ & $50.5 \pm 13.9$ & 2,382 & 0.109 & & 0.001 & $46.3 \pm 14.8$ & $47.5 \pm 15.0$ & $49.3 \pm 18.7$ & 2,225 & 0.591 & & 0.005 \\
\hline Fouls & $14.2 \pm 3.8$ & $13.7 \pm 4.7$ & $13.4 \pm 4.8$ & 2,176 & 0.298 & & 0.003 & $15.0 \pm 4.2$ & $13.5 \pm 4.4$ & $14.8 \pm 4.1$ & 2,382 & 4.510 & $*$ & 0.023 & $15.6 \pm 4.7$ & $14.2 \pm 4.0$ & $15.7 \pm 4.7$ & 2,225 & 2.371 & & 0.021 \\
\hline Tackles & $19.9 \pm 5.8$ & $20.6 \pm 3.8$ & $21.6 \pm 6.4$ & 2,176 & 1.220 & & 0.014 & $21.5 \pm 5.8$ & $21.0 \pm 5.7$ & $21.4 \pm 6.3$ & 2,382 & 0.231 & & 0.001 & $20.7 \pm 6.4$ & $21.5 \pm 5.5$ & $21.4 \pm 5.2$ & 2,225 & 0.457 & & 0.004 \\
\hline $\mathrm{YC}$ & $2.3 \pm 1.4$ & $2.1 \pm 1.4$ & $1.6 \pm 1.2$ & 2,176 & 4.279 & * & 0.046 & $2.1 \pm 1.3$ & $2.0 \pm 1.2$ & $1.7 \pm 1.2$ & 2,382 & 3.479 & * & 0.018 & $2.2 \pm 1.3$ & $2.0 \pm 1.2$ & $2.2 \pm 1.1$ & 2,225 & 0.699 & & 0.006 \\
\hline
\end{tabular}

Note. Median, lower and upper quartiles were removed due to table size limitation 
High Level Teams

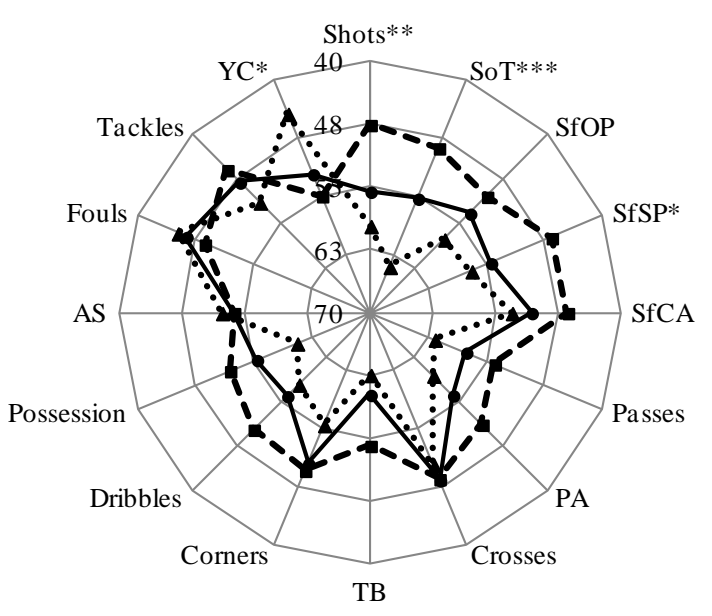

Intermediate Level Teams

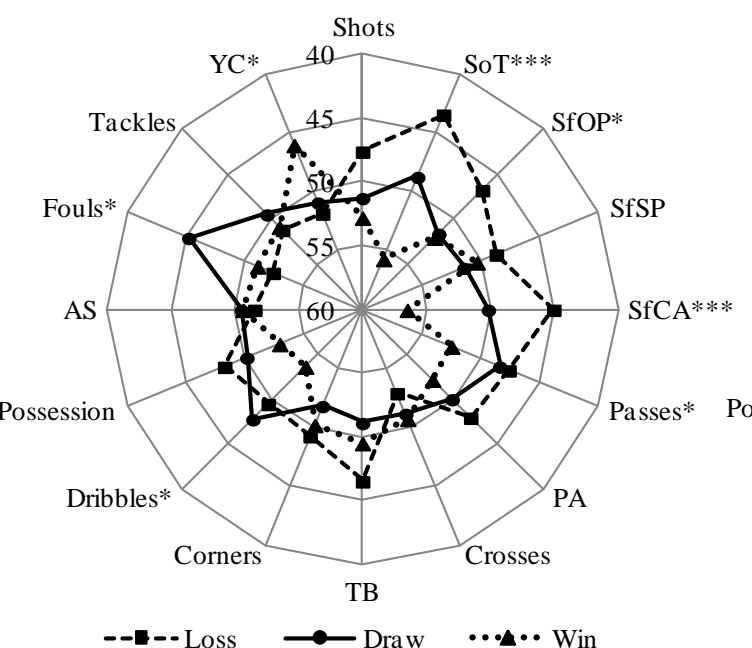

Low Level Teams

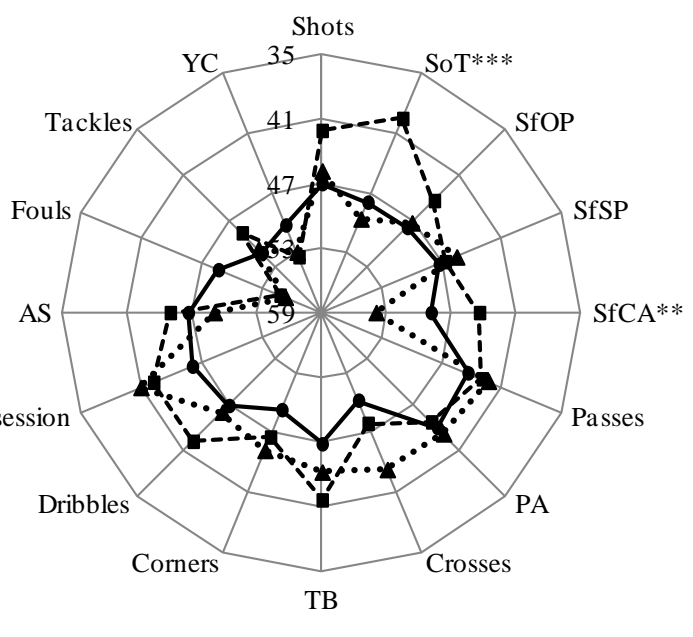

Figure 3. Comparison on performances of high, intermediate and low level teams when ending with different match outcomes 
It can be seen from performance profiles when ending with different match outcomes that various variables were found significantly different from won, drawn and lost matches for all three levels of team in UEFA Champions League, which is in accordance to prior research in Spanish First Division (Lago-Peñas et al., 2010), World Cup (Castellano et al., 2012) and Group Stage of UEFA Champions League (Lago-Peñas et al., 2011). Surprisingly, only three performance-related variables (shots, shots on target and through balls) were found to be changed by different match outcomes for high, intermediate and low level teams. Meanwhile, it is worthwhile to note that for the low-level teams, their winner games showed higher values comparing to drawn and lost games in shots, shots on target, shots from counter attack, through balls and aerial success, while other variables were not significantly different. Coaches and players of low-level teams could adopt appropriate tactics depending on this fact; meanwhile, these of intermediate and high level teams need to take suitable countering measures when facing low-level teams.

\subsection{Performance profiles when playing at different locations}

Profiles of performance of teams of different levels of strength when playing at home and away were shown in Table 4 . While Figure 4 compared the mean differences of all performance-related match variables of all three levels of team playing at different locations. 
Table 4. Profiles of performance of high, intermediate and low-level teams when playing at different locations

\begin{tabular}{|c|c|c|c|c|c|c|c|c|c|c|c|c|c|c|c|c|c|c|c|c|c|}
\hline \multirow{3}{*}{ Variables } & \multicolumn{7}{|c|}{ High-level teams } & \multicolumn{7}{|c|}{ Intermediate-level teams } & \multicolumn{7}{|c|}{ Low-level teams } \\
\hline & Home & Away & & & & & & Home & Away & & & & & & Home & Away & & & & & \\
\hline & Mean \pm SD & Mean \pm SD & $t$ & df & Sig. & ES & $95 \%$ CI & Mean \pm SD & Mean \pm SD & $\mathrm{t}$ & df & Sig. & ES & $95 \%$ CI & Mean \pm SD & Mean \pm SD & $\mathrm{t}$ & df & Sig. & ES & $95 \% \mathrm{CI}$ \\
\hline Shots & $17.6 \pm 6.1$ & $13.6 \pm 5.5$ & 4.549 & 172 & $* * *$ & 0.63 & \pm 1.7 & $15.4 \pm 6.1$ & $12.2 \pm 5.3$ & 5.541 & 383 & $* * *$ & 0.53 & \pm 1.1 & $13.7 \pm 6.3$ & $10.0 \pm 4.7$ & 5.033 & 227 & $* * *$ & 0.63 & \pm 1.4 \\
\hline SoT & $6.4 \pm 2.8$ & $5.2 \pm 2.7$ & 3.054 & 172 & $* *$ & 0.41 & \pm 0.8 & $5.2 \pm 2.5$ & $4.1 \pm 2.3$ & 4.758 & 383 & $* * *$ & 0.46 & \pm 0.5 & $4.3 \pm 2.5$ & $3.4 \pm 2.1$ & 3.030 & 227 & $* *$ & 0.28 & \pm 0.6 \\
\hline SfOP & $10.9 \pm 5.3$ & $8.6 \pm 4.5$ & 3.163 & 173 & $* *$ & 0.54 & \pm 1.5 & $9.6 \pm 4.3$ & $7.6 \pm 4.2$ & 4.759 & 387 & $* * *$ & 0.45 & \pm 0.9 & $8.8 \pm 4.9$ & $6.4 \pm 3.7$ & 4.107 & 226 & $* * *$ & 0.52 & \pm 1.1 \\
\hline SfSP & $4.1 \pm 2.2$ & $2.9 \pm 2.0$ & 3.762 & 173 & $* * *$ & 0.42 & \pm 0.6 & $3.4 \pm 2.3$ & $2.6 \pm 1.8$ & 3.758 & 387 & $* * *$ & 0.44 & \pm 0.4 & $3.0 \pm 2.3$ & $2.3 \pm 1.6$ & 2.515 & 226 & $*$ & 0.35 & \pm 0.5 \\
\hline SfCA & $0.5 \pm 0.8$ & $0.5 \pm 0.8$ & 0.286 & 173 & & 0.00 & \pm 0.2 & $0.5 \pm 0.8$ & $0.6 \pm 0.8$ & 1.026 & 387 & & 0.18 & \pm 0.2 & $0.4 \pm 0.7$ & $0.4 \pm 0.7$ & 0.117 & 226 & & 0.31 & \pm 0.2 \\
\hline Passes & $553.7 \pm 165.7$ & $529.2 \pm 171.9$ & 0.957 & 174 & & 0.16 & \pm 50.5 & $493.0 \pm 110.3$ & $465.3 \pm 109.3$ & 2.490 & 387 & * & 0.26 & \pm 21.9 & $454.9 \pm 104.7$ & $420.7 \pm 94.4$ & 2.594 & 227 & * & 0.32 & \pm 26.0 \\
\hline $\mathrm{PA}$ & $82.1 \pm 6.9$ & $80.8 \pm 8.0$ & 1.104 & 172 & & 0.18 & \pm 2.2 & $79.9 \pm 6.3$ & $78.3 \pm 6.4$ & 2.485 & 383 & $*$ & 0.25 & \pm 1.3 & $77.9 \pm 6.8$ & $76.0 \pm 7.5$ & 1.975 & 227 & $*$ & 0.25 & \pm 1.9 \\
\hline Crosses & $22.9 \pm 9.0$ & $18.5 \pm 8.5$ & 3.282 & 174 & $* *$ & 0.46 & \pm 2.6 & $23.9 \pm 8.7$ & $18.5 \pm 8.4$ & 6.215 & 387 & $* * *$ & 0.67 & \pm 1.7 & $23.3 \pm 9.6$ & $15.9 \pm 7.5$ & 6.482 & 227 & $* * *$ & 0.80 & \pm 2.2 \\
\hline TB & $5.4 \pm 4.6$ & $4.8 \pm 4.1$ & 0.841 & 174 & & 0.03 & \pm 1.3 & $3.4 \pm 2.8$ & $3.1 \pm 2.8$ & 1.142 & 387 & & 0.14 & \pm 0.6 & $2.2 \pm 2.4$ & $2.5 \pm 2.4$ & 1.008 & 227 & & 0.17 & \pm 0.6 \\
\hline Corners & $6.3 \pm 3.1$ & $5.0 \pm 2.9$ & 2.935 & 172 & $* *$ & 0.43 & \pm 0.9 & $5.8 \pm 3.0$ & $4.5 \pm 2.8$ & 4.214 & 383 & $* * *$ & 0.48 & \pm 0.6 & $5.2 \pm 2.8$ & $4.1 \pm 2.8$ & 3.091 & 227 & $* *$ & 0.34 & \pm 0.7 \\
\hline Dribbles & $9.7 \pm 4.8$ & $8.5 \pm 3.6$ & 1.927 & 172 & & 0.25 & \pm 1.3 & $8.6 \pm 4.1$ & $7.6 \pm 3.8$ & 2.482 & 383 & * & 0.22 & \pm 0.8 & $6.7 \pm 3.8$ & $6.8 \pm 3.4$ & 0.142 & 227 & & 0.09 & \pm 0.9 \\
\hline Possession & $56.2 \pm 14.3$ & $53.1 \pm 14.3$ & 1.416 & 172 & & 0.20 & \pm 4.3 & $51.7 \pm 10.4$ & $49.1 \pm 10.6$ & 2.367 & 383 & $*$ & 0.23 & \pm 2.1 & $48.0 \pm 11.3$ & $43.6 \pm 10.1$ & 3.092 & 227 & $* *$ & 0.36 & \pm 2.8 \\
\hline AS & $52.0 \pm 14.1$ & $52.5 \pm 15.0$ & 0.265 & 172 & & 0.02 & \pm 4.4 & $50.7 \pm 14.7$ & $50.6 \pm 13.3$ & 0.090 & 383 & & 0.02 & \pm 2.8 & $48.4 \pm 15.1$ & $45.9 \pm 15.9$ & 1.243 & 227 & & 0.19 & \pm 4.0 \\
\hline Fouls & $13.0 \pm 4.7$ & $14.4 \pm 4.4$ & 1.978 & 172 & * & 0.35 & \pm 1.4 & $14.0 \pm 3.9$ & $15.0 \pm 4.5$ & 2.312 & 383 & * & 0.17 & \pm 0.8 & $14.7 \pm 4.2$ & $15.7 \pm 4.8$ & 1.591 & 227 & & 0.20 & \pm 1.2 \\
\hline Tackles & $20.9 \pm 6.7$ & $21.0 \pm 5.8$ & 0.070 & 172 & & 0.07 & \pm 1.9 & $21.6 \pm 6.2$ & $21.1 \pm 5.7$ & 0.853 & 383 & & 0.06 & \pm 1.2 & $20.2 \pm 5.7$ & $21.9 \pm 6.0$ & 2.160 & 227 & $*$ & 0.26 & \pm 1.5 \\
\hline $\mathrm{YC}$ & $1.5 \pm 1.1$ & $2.3 \pm 1.4$ & 4.112 & 174 & $* * *$ & 0.54 & \pm 0.4 & $1.7 \pm 1.2$ & $2.1 \pm 1.2$ & 3.331 & 387 & $* * *$ & 0.18 & \pm 0.2 & $2.0 \pm 1.4$ & $2.2 \pm 1.1$ & 1.503 & 227 & & 0.09 & \pm 0.3 \\
\hline
\end{tabular}

Note 1. ES = Effect Size, 95\% CI = 95\% Confidence Interval of the Difference in Mean

Note 2. Median, lower and upper quartiles were removed due to table size limitation 

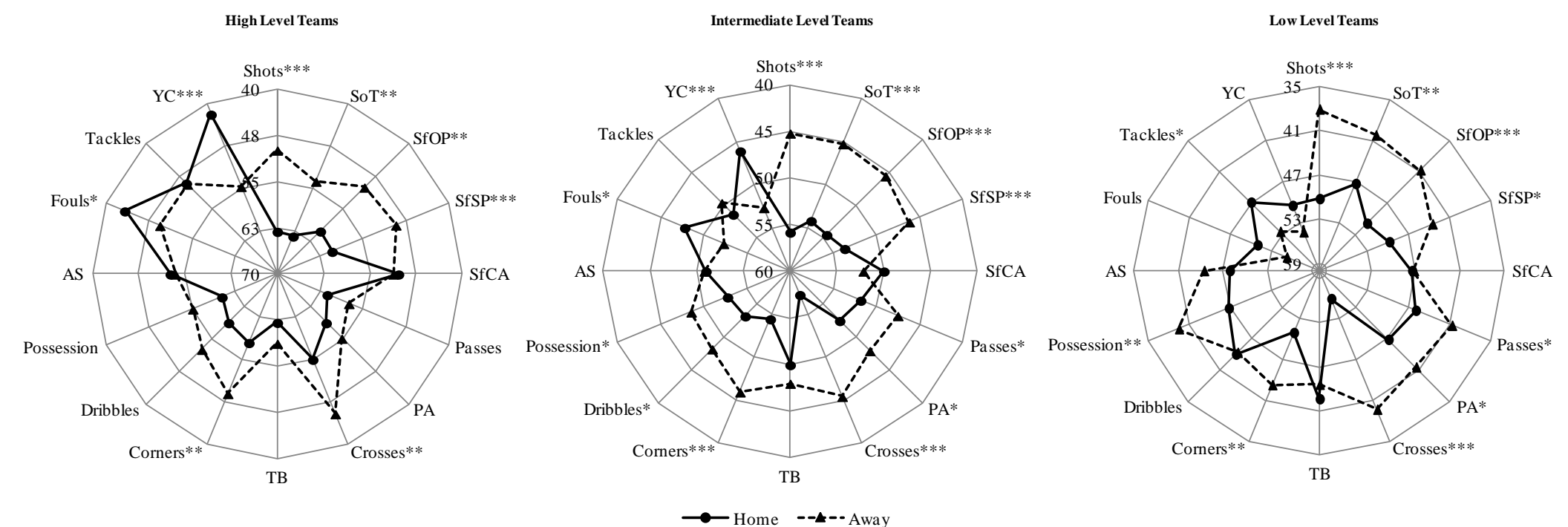

Figure 4. Comparison on performances of high, intermediate and low level teams when playing at home and away 
Performance profiles playing at different locations showed that almost all variables differed when playing at home and away for all three levels of team which agreed to results of previous studies on Spanish First Division (Gómez et al., 2012; Lago-Peñas and Lago-Ballesteros, 2011; Lago, 2009) and England Premier League (Taylor et al., 2010; Taylor et al., 2008). The two variables that were not significantly different for all levels of team were shots from counter attack and through balls, which could be due to their relatively small numbers. Our results also showed that high-level teams' home and away differences mainly appeared in the variables related to scoring and defending; however, significant differences were observed in most of variables for intermediate- and low-level teams. It may be the reason that, comparing to inferior teams, high-level teams tend to employ more pro-active defensive strategies and they apply more actions aiming directly to recovery the ball from opponent's half of pitch which may lead to more chances of scoring (Almeida et al., 2014). As well, these findings confirmed the conclusion of Lago-Peñas and Lago-Ballesteros (2011, p. 470) that indicated "teams described as superior and those described as inferior did not experience the same home advantage", which is to say that high-level teams' technical and tactical performance is more stable either playing home or away than the intermediate and low level teams.

By comparisons on performance profiles of teams of all three levels of strength, it can be found that high-level teams are characterised by stability of performance no matter play against which opponent, with result pressure or where they play. On the other hand, performances of intermediate- and low-level teams are associated with more variation to different situational variables.

Apart from the comparisons, typical performances and spreads of different levels of team playing in different situations were identified by the profiles as well. These could provide some information and assistance to coaches and analysts when analysing the strength and weakness of their opponents and of their own in a specific way (O'Donoghue, 2013). For example, when a relatively weaker team is going to play at home against a relatively much stronger opponent, the typical performance of high-level team playing away against low-level team can provide advanced references on the upcoming opponent's strategies. Contrarily, typical performance of low-level team playing at home against high-level team could be references for the opponent.

The detailed evaluation and comparison of the influence of strength of opposition, match location and match outcome on football performance using profiling techniques within this study presents a number of implications for analysts and coaches. Existing recommendations suggest that the scouting of upcoming opposition should be carried out under circumstances that are reflective of the conditions under which the future match will occur. However, such procedures are unlikely to be practical due to time and resource constraints. Consequently, by establishing profiles of impact of particular situational variable on performances, oppositions can be observed, or at least, appropriate observing aspects on analysing the upcoming opponent based on the 
knowledge of such effects could be provided. Similarly, post-match assessments of performance on the own team can be made more objectively and directly by profiling performance-related match variables in effects of situational variables. Finally, variation of teams' performance associated with specific situational variables could be identified by the profiles, hence, possible causes can be examined and match preparation focusing on reducing such effects can be made.

\section{Limitations}

Although this study has considered the situational variables at a behavioral level in a great depth, there are several limitations that provide subsequent directions for future research. Firstly, the interactive effects of situational variables on team performance were not interpreted. Secondly, the stage and period (e.g. group stage and knockout stage) of the competition may affect the result and performance which was not included either. Future studies of this kind should take these aspects into account.

\section{Acknowledgements}

Authors acknowledge the financial support from the Spain Ministry of Science and Innovation, research project DEP2011-23338. The first author is funded by the China Scholarship Council (CSC) from the Ministry of Education of PR. China.

\section{References}

Almeida, C. H., Ferreira, A. P., and Volossovitch, A. (2014), Effects of Match Location, Match Status and Quality of Opposition on Regaining Possession in UEFA Champions League, Journal of Human Kinetics, 41, 203-214.

Armatas, V., Yiannakos, A., Zaggelidis, G., Skoufas, D., Papadopoulou, S., and Fragkos, N. (2009), Goal scoring patterns in Greek top leveled football matches, Journal of Physical Education and Sport, 23, 1-5.

Barriopedro, M. I. F., and Muniesa, C. (2012), Analisis de Datos en Las Ciencias de La Actividad Fisica y Del Deporte (Data Analysis in the Sciences of Physical Activity and Sport). Madrid: Piramide, pp. 60-64.

Butterworth, A., O'Donoghue, P., and Cropley, B. (2013), Performance profiling in sports coaching: a review, International Journal of Performance Analysis in Sport, 13, 572-593.

Castellano, J., Casamichana, D., and Lago, C. (2012), The Use of Match Statistics that Discriminate Between Successful and Unsuccessful Soccer Teams, Journal of Human Kinetics, 31, 139-147.

Cohen, J. (1988), Statistical power analysis for the behavioral sciences (2nd ed.). Hillsdale, NJ: Erlbaum, pp. 273-288. 
Eugster, M. J. A. (2012), Performance Profiles based on Archetypal Athletes, International Journal of Performance Analysis in Sport, 12, 166-187.

Gómez, M. A., Lago-Peñas, C., and Pollard, R. (2013). Situational Variables. In T. McGarry, P. O'Donoghue \& J. Sampaio (Eds.), Routledge Handbook of Sports Performance Analysis (pp. 259-269). London: Routledge.

Gómez, M. A., DelaSerna, A., Lupo, C., and Sampaio, J. (2014), Effects of Situational Variables and Starting Quarter Score in the outcome of elite women's water polo game quarters, International Journal of Performance Analysis in Sport, 14, 73-83.

Gómez, M. A., Gómez-Lopez, M., Lago, C., and Sampaio, J. (2012), Effects of game location and final outcome on game-related statistics in each zone of the pitch in professional football, European Journal of Sport Science, 12, 393-398.

Hopkins, W. G. (2007). A spreadhseet to compare means of two groups. Retrieved 12 January, 2015, from http://www.sportsci.org/2007/inbrief.htm\#xcl2

Hopkins, W. G., Marshall, S. W., Batterham, A. M., and Hanin, J. (2009), Progressive statistics for studies in sports medicine and exercise science, Medicine and Science in Sports and Exercise, 41, 3-13.

Hughes, M. D., Evans, S., and Wells, J. (2001), Establishing normative profiles in performance analysis, International Journal of Performance Analysis in Sport, 1, 1-26.

James, N., Mellalieu, S. D., and Jones, N. M. P. (2005), The development of position-specific performance indicators in professional rugby union, Journal of Sports Sciences, 23, 63-72.

Lago-Peñas, C. (2012), The role of situational variables in analysing physical performance in soccer, Journal of Human Kinetics, 35, 89-95.

Lago-Peñas, C., and Lago-Ballesteros, J. (2011), Game location and team quality effects on performance profiles in professional soccer, Journal of Sports Science and Medicine, 10, 465-471.

Lago-Peñas, C., Lago-Ballesteros, J., Dellal, A., and Gómez, M. (2010), Game-Related Statistics that Discriminated Winning, Drawing and Losing Teams from the Spanish Soccer League, Journal of Sports Science and Medicine, 9, 288-293.

Lago-Peñas, C., Lago-Ballesteros, J., and Rey, E. (2011), Differences in Performance Indicators between Winning and Losing Teams in the UEFA Champions League, Journal of Human Kinetics, 27, 137-148.

Lago, C. (2009), The influence of match location, quality of opposition, and match status on possession strategies in professional association football, Journal of Sports Sciences, 27, 1463-1469.

Liu, H., Hopkins, W., Gómez, M. A., and Molinuevo, J. S. (2013), Inter-operator reliability of live football match statistics from OPTA Sportsdata, International Journal of Performance Analysis in Sport, 13, 803-821.

Lupo, C., Condello, G., Capranica, L., and Tessitore, A. (2014), Women's Water Polo World Championships: Technical and Tactical Aspects of Winning and Losing Teams in Close and Unbalanced Games, Journal of Strength and 
Conditioning Research, 28, 210-222.

Mackenzie, R., and Cushion, C. (2013), Performance analysis in football: A critical review and implications for future research, Journal of Sports Sciences, 31, 639-676.

Marcelino, R., Mesquita, I., and Sampaio, J. (2011), Effects of quality of opposition and match status on technical and tactical performances in elite volleyball, Journal of Sports Sciences, 29, 733-741.

O'Donoghue, P. (2005), Normative profiles of sports performance, International Journal of Performance Analysis in Sport, 5, 104-119.

O’Donoghue, P. (2013). Sports performance profiling. In T. McGarry, P. O’Donoghue \& J. Sampaio (Eds.), Routledge Handbook of sports performance analysis (pp. 127-139). London: Routledge.

Oberstone, J. (2009), Differentiating the top English premier league football clubs from the rest of the pack: Identifying the keys to success, Journal of Quantitative Analysis in Sports, 5

Sampaio, J., Lago, C., and Drinkwater, E. J. (2010), Explanations for the United States of America's dominance in basketball at the Beijing Olympic Games (2008), Journal of Sports Sciences, 28, 147-152.

Sarmento, H., Marcelino, R., Anguera, M. T., Campanico, J., Matos, N., and Leitao, J. C. (2014), Match analysis in football: a systematic review, Journal of Sports Sciences, 32, 1831-1843.

Taylor, J. B., Mellalieu, S. D., James, N., and Barter, P. (2010), Situation variable effects and tactical performance in professional association football, International Journal of Performance Analysis in Sport, 10, 255-269.

Taylor, J. B., Mellalieu, S. D., James, N., and Shearer, D. A. (2008), The influence of match location, quality of opposition, and match status on technical performance in professional association football, Journal of Sports Sciences, 26, 885-895.

UEFA. (2013). UEFA Rankings - UEFA.com Retrieved 12 Oct, 2013

Vilar, L., Araujo, D., Davids, K., and Button, C. (2012), The Role of Ecological Dynamics in Analysing Performance in Team Sports, Sports Medicine, 42, $1-10$. 